\title{
Line profiles of OB star winds using a Monte Carlo method
}

\author{
Brankica Šurlan $^{1,2}$ and Jiří Kubát ${ }^{1}$ \\ ${ }^{1}$ Astronomický ústav, Akademie věd České republiky, CZ-251 65 Ondřejov \\ email: surlan, kubat@sunstel.asu.cas.cz \\ ${ }^{2}$ Matematički Institut SANU, Kneza Mihaila 36, 11001 Beograd, Serbia
}

\begin{abstract}
The solution of the radiative transfer in an expanding atmospheres using the Monte Carlo method is presented. We applied our method to winds of several OB stars. In our calculation, the velocity and density structure is assumed to be given. Selected line profiles are shown.
\end{abstract}

Keywords. radiative transfer, line: formation, stars: winds, outflows

\section{Introduction}

We started to develop a code for the solution of the radiative transfer problem which could be used for the solution of line formation in a 3-D inhomogeneous medium. The code is based on a Monte Carlo method. As a first step, we developed a spherically symmetric code for the formal solution of the radiative transfer equation, i.e. for a solution of this equation for given velocity, temperature, and density stratification. This task has already been done by many authors for different astrophysical applications, e.g. Abbott \& Lucy (1985), Whitney (1991), Lucy \& Abbott (1993), de Koter et al. (1997), Vink et al. (1999), Carciofi \& Bjorkman (2008), Wood et al. (2004), and Kromer \& Sim (2009), to name at least some of them. Nevertheless, in order to understand all processes more deeply and to have better control of the code's behaviour, we decided to develop an independent code. Its detailed description will be published elsewhere.

\section{Wind model and results}

The velocity structure $v(r)$ of the wind is calculated using the $\beta$-velocity law. Knowing the velocity structure and the mass-loss rate $\dot{M}$, the density structure $\rho(r)$ follows from the continuity equation. The temperature $T(r)$ was taken as independent of $r$ and equals the approximate value of the radiation temperature $T_{\text {rad }} \approx 3 / 4 T_{\text {eff }}$. This value was used for calculation of the LTE ionization and excitation equilibrium in the wind, while the electron density was calculated consistently. We further assume that all electrons in the wind come from hydrogen ionization, and that the opacity of the medium consists of only line scattering under the Sobolev approximation. For depth discretization we used equidistant spacing in the logarithmic scale. Depth points split the wind into zones. The density $\rho(r)$ was taken to be constant within a zone equal to the value at the lower radius of the zone. The radial velocity $v(r)$ is linearly interpolated inside the zone. The flux at the lower boundary of the wind was computed as an emergent flux from the static spherically symmetric NLTE model atmosphere calculated using a code of Kubát (2003). Parameters for selected stars were taken from Crowther et al. (2006) and are listed in Table 1. 
Table 1. Stellar and wind parameters taken from Crowther et al. (2006).

\begin{tabular}{|c|c|c|c|r|c|c|}
\hline Name & $\begin{array}{c}T_{\text {eff }} \\
{[\mathrm{K}]}\end{array}$ & $\log g$ & $\begin{array}{c}R_{*} \\
{\left[R_{\odot}\right]}\end{array}$ & $\begin{array}{c}v_{\infty} \\
{[\mathrm{km} / \mathrm{s}]}\end{array}$ & $\begin{array}{c}\dot{M} \\
{\left[10^{-6} M_{\odot} / y r\right]}\end{array}$ & $\beta$ \\
\hline HD 14818 & 18500 & 2.40 & 46.1 & 565 & 0.55 & 2.0 \\
HD 13854 & 21500 & 2.55 & 37.4 & 920 & 0.85 & 2.0 \\
HD 115842 & 25500 & 2.85 & 34.2 & 1180 & 2.00 & 1.5 \\
HD 30614 & 29000 & 3.00 & 26.0 & 1560 & 5.00 & 1.5 \\
\hline
\end{tabular}
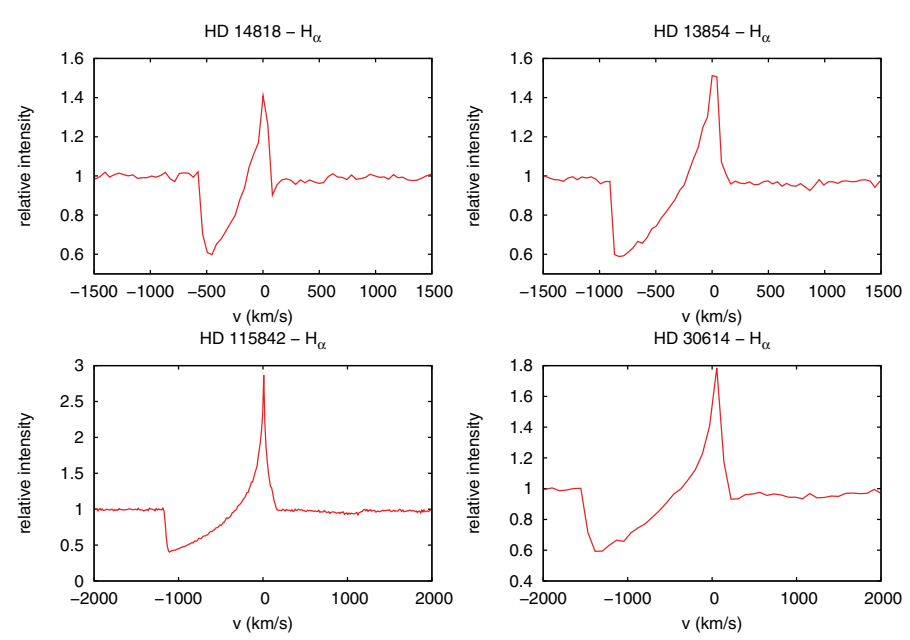

Figure 1. $\mathrm{H} \alpha$ profiles for stars from Table 1.

We plot the profiles of the $\mathrm{H} \alpha$ line of four OB stars obtained as a result of our calculation using Monte Carlo radiative transfer. Stellar and wind parameters are given in Table 1. The flux is expressed as relative intensity with respect to the local continuum. The terminal velocities, which follow from the theoretical profiles, correspond well to measured terminal velocities of the wind $\left(v_{\infty}\right.$ in Table 1$)$.

Our code is able to solve the line formation problem in an expanding medium for the spherically symmetric case. In our future work we aim at generalizing the code to 3D and including inhomogeneities.

\section{Acknowledgements}

This research was supported by GA ČR grants 205/08/0003 and 205/08/H005.

\section{References}

Abbott, D. C. \& Lucy, L. B. 1985, ApJ 288, 679

Carciofi, A. C. \& Bjorkman, J. E. 2008, ApJ 684, 1374

Crowther, P. A., Lennon, D. J., \& Walborn, N. R. 2006, A\& A 446, 279

de Koter, A., Heap, S. R., \& Hubeny, I. 1997, ApJ 477, 792

Kromer, M. \& Sim, S. A. 2009, MNRAS 398, 1809

Kubát, J. 2003, in: N. Piskunov, W. W. Weiss, \& D. F. Gray (eds.), Modelling of Stellar Atmospheres, IAU Symposium 210, p. $6 \mathrm{P}$

Lucy, L. B. \& Abbott, D. C. 1993, ApJ 405, 738

Whitney, B. A. 1991, ApJS 75, 1293

Wood, K., Mathis, J. S., \& Ercolano, B. 2004, MNRAS 348, 1337

Vink, J. S., de Koter, A., \& Lamers, H. J. G. L. M. 1999, A\& A 350, 181 